\title{
CONTROLLING STEADY-STATE AND DYNAMICAL PROPERTIES OF ATOMIC OPTICAL BISTABILITY
}


This page intentionally left blank 


\section{CONTROLLING STEADY-STATE AND DYNAMICAL PROPERTIES OF ATOMIC OPTICAL BISTABILITY}
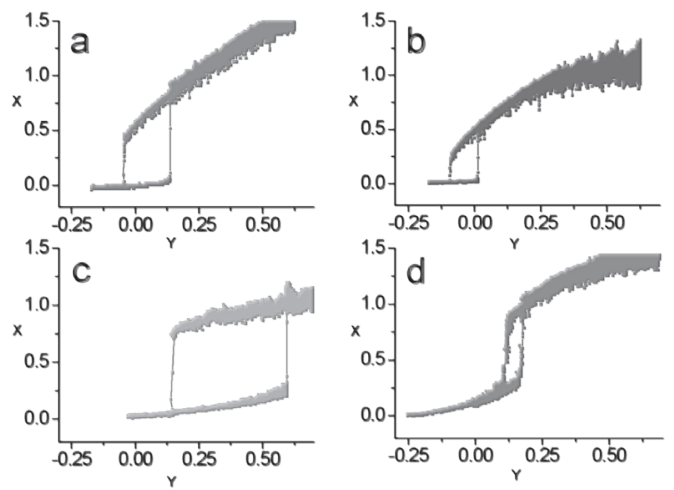

\section{Amitabh Joshi}

Eastern Illinois University, USA

Min Xiao

University of Arkansas, USA 
Published by

World Scientific Publishing Co. Pte. Ltd.

5 Toh Tuck Link, Singapore 596224

USA office: 27 Warren Street, Suite 401-402, Hackensack, NJ 07601

UK office: 57 Shelton Street, Covent Garden, London WC2H 9HE

\section{British Library Cataloguing-in-Publication Data}

A catalogue record for this book is available from the British Library.

\section{CONTROLLING STEADY-STATE AND DYNAMICAL PROPERTIES OF ATOMIC OPTICAL BISTABILITY}

Copyright () 2012 by World Scientific Publishing Co. Pte. Ltd.

All rights reserved. This book, or parts thereof, may not be reproduced in any form or by any means, electronic or mechanical, including photocopying, recording or any information storage and retrieval system now known or to be invented, without written permission from the Publisher.

For photocopying of material in this volume, please pay a copying fee through the Copyright Clearance Center, Inc., 222 Rosewood Drive, Danvers, MA 01923, USA. In this case permission to photocopy is not required from the publisher.

ISBN-13 978-981-4307-55-0

ISBN-10 981-4307-55-6

Printed in Singapore. 
We dedicate this book to our parents 
This page intentionally left blank 


\section{Preface}

Atomic optical bistability (AOB) has been a very active field of research in eighties because of its potential application in all-optical logic and switching devices. Bistability was first observed in a passive atomic medium of sodium vapor in 1974 and since then a wide range of activities started in this field of research, and many nonlinear materials including semiconductors were investigated for bistability. The main research was centered on optimizing the size of devices, switching times, operating powers, operating temperatures and other operating conditions. There have been a comprehensive book and some review articles for AOB in two-level systems, such as the excellent book by H. Gibbs: Optical Bistability: Controlling light with light (Academic Press, 1985) and a review article by L. A. Lugiato: Theory of optical bistability in 'Progress in Optics', Vol. 21, p.71 (edited by E. Wolf, North Holland, Amsterdam, 1984). Also, the phenomenon of optical bistability has been discussed in several text books, such as 'Nonlinear Optics' by R. W. Boyd (Elsevier, 3rd Edition, 2008).

In the decade of nineties a great deal of research activities have been seen in the area of multilevel atomic systems exhibiting the phenomenon of electromagnetically induced transparency (EIT). The multilevel atomic system has several advantages over the two-level system as absorption, dispersion, and nonlinear optical properties of one optical field coupled to one atomic transition can be greatly modified by another optical fields coupled to nearby atomic transitions due to the induced atomic coherences. By using such changes in linear and nonlinear optical properties around resonance, due to the EIT, it is easy to manipulate and control nonlinear optical processes in the multilevel atomic systems. Such controls are not available in the two-level atomic system, which have stimulated new research interests in the area of AOB using multilevel atomic systems inside the optical 
cavity. Hence, one of the new research areas is the controllability of AOB in a three-level atomic system inside an optical cavity, emphasizing the enhanced nonlinear optical process due to induced atomic coherence in such intracavity EIT medium. All-optical switching using AOB and dynamical hysteresis in $\mathrm{AOB}$ were studied using the three-level atomic system. Investigations of controlled optical dynamic instability and chaos in the cavity output field with three-level atoms inside an optical cavity were carried out. Phenomenon of stochastic resonance in $\mathrm{AOB}$ and noise-induced transitions became easily accessible experiments because of the enhanced nonlinearity in the intracavity EIT medium. Some of those effects have not been observed previously in two-level AOB systems.

The book presents the descriptions of unique controllabilities available in the multilevel EIT systems for studying AOB and related effects, along with the underlying physics encompassing those phenomena. The book is aimed at both graduate level courses and beginning researchers in these areas of $\mathrm{AOB}$ and related effects. It can also serve as a reference book for many interesting aspects of nonlinear optical phenomena. We first introduce the background materials for two- and three-level atomic systems useful in understanding optical bistability, multistabilities, dynamic instability, chaos, stochastic resonance, and all-optical as well as noise-induced switching. The book is written at a level comprehensible to readers with the background of a beginning graduate student in the disciplines related to physics and electrical engineering having some good understanding in mathematics, and electricity-magnetism. The other purpose of the book is to develop in detail the central results of optical bistability and associated phenomena with multilevel atomic media inside optical cavity. Through studying this book, the reader should develop a working understanding of fundamental tools and results of this exciting field, and be able to carry out independent research in areas related to $\mathrm{AOB}$ and other nonlinear optical processes in multilevel systems.

The basic structure of the book is as follows. Chapter 1 gives an introduction and fundamental concepts of interaction of electromagnetic field with two- and three-level atomic systems, electrical susceptibilities, nonlinearities of these systems, highlighting some open issues along the way. A broad overview for the main concepts and results of multilevel EIT systems, including controllable linear absorption and dispersion properties in threelevel atomic systems are presented. The in-depth fundamental notions of AOB along with the historical development for the simple case of two-level atoms are reviewed in Chapter 2. In Chapter 3 the discussion on cavity line- 
narrowing effects and cavity ring down spectroscopy with three-level EIT systems are given. This Chapter also provides formulation of steady-state AOB with three-level atoms inside an optical cavity, which is followed by descriptions of recent experiments showing controls of shape and rotation direction of the hysteresis cycles by the coupling beam frequency detuning and other experimental parameters. The background material and origin of optical multistability in two- and three-level atoms in terms of the general principle are discussed in Chapter 4. The discussion is further enhanced by incorporating some successful experimental demonstrations of optical multistability in the laboratory.

Chapter 5 describes theoretical and experimental investigations of controlled optical dynamic instability and chaos in the cavity output field with three-level atoms inside an optical ring cavity. The route to chaos via period doubling and its characterization with the help of diagnostic recipes of Fourier transform and Lyapunov exponent calculations are discussed.

In Chapter 6, experiments describing deterministic switching are discussed. The basics of all-optical switching phenomena and their controls using Kerr nonlinearity are described, which were demonstrated in the basic three-level AOB system inside an optical cavity. In such experiments the cavity (probe) output field intensity could be controlled by the coupling field intensity or frequency detuning. The importance of such all-optical switching in optical computation is also highlighted.

The dynamical hysteresis, stochastic resonance and noise-induced transitions in the three-level AOB system are explained in Chapter 7. The formulations of these phenomena, both qualitatively and with simplified mathematical models, are presented in this Chapter. Then, a few novel experiments done in laboratory are discussed.

Some concluding remarks and outlooks are given in Chapter 8 which summarizes this book with emphasis on the unified central theme: controllable cavity output (both steady-state and dynamical behaviors) with a modified linear and nonlinear intracavity medium of an ensemble of threelevel EIT atoms.

We would like to thank many colleagues who have worked with us over the years on the topics presented in this book, especially H. Wang, D. J. Goorskey, W. Yang, and A. Brown. We also thank our families for their supports and understanding. 
This page intentionally left blank 


\section{Contents}

Preface vii

1. Introduction 1

1.1 Background . . . . . . . . . . . . . . . 1

1.2 Nonlinearity in a Two-level Atomic System . . . . . . . . 6

1.2.1 Two-level atoms interacting with a monochromatic field: the density-matrix approach . . . . . . . 6

1.2.2 Absorption and dispersion spectra in steady-state 10

1.2.3 First- and third-order susceptibilities and the saturation phenomenon . . . . . . . . . . 12

1.3 Doppler Effect in Inhomogeneously-broadened Atomic Systems ......................... 13

1.3.1 Doppler effect in a two-level atomic system . . . . 14

1.3.2 Doppler effect in three-level atomic systems . . . . 16

1.4 Optical Cavity . . . . . . . . . . . . . . . 17

1.4.1 Optical Fabry-Perot cavity: transmission and reflection functions, finesse and quality factor . . . . 18

1.4.2 Optical ring cavity . . . . . . . . . . . . . 24

1.4.3 Optical ring cavity with an intracavity medium: modification of transmission function . . . . . . 24

1.5 Controllable Linear and Nonlinear Susceptibilities in Three-level Atomic Systems . . . . . . . . . . . . . . 26

1.5.1 Early works on coherent population trapping . . 27

1.5.2 Coherent population trapping vs electromagnetically induced transparency . . . . . . . . . . . 28 
1.5.3 Controlling linear absorption and dispersion properties in three-level electromagnetically induced transparency systems . . . . . . . . . . .

1.5.4 Enhancement and control of Kerr nonlinearity in three-level electromagnetically induced transparency systems . . . . . . . . . . .

2. Atomic Optical Bistability in a Two-level System

2.1 Two-level Atoms inside an Optical Cavity . . . . . . . . .

2.1.1 Split in transmission spectrum of the optical cavity . . . . . . . . . . . . . 52

2.2 Atomic Optical Bistability _. . . . . . . . . 55

2.2.1 Atomic optical bistability: the mean field theory . $\quad 56$

2.3 Absorptive Atomic Optical Bistability . . . . . . . . . 57

2.3.1 Simple model of absorptive atomic optical bistability ................ 57

2.3.2 Mean field theory of absorptive atomic optical bistability . . . . . . . . . . . 60

2.4 Dispersive/refractive Atomic Optical Bistability . . . . . . 62

2.4.1 Simple model of dispersive/refractive atomic optical bistability . . . . . . . . . . . .

2.4.2 Mean field theory of dispersive/refractive atomic optical bistability . . . . . . . . . . 63

2.5 Mixed Absorptive-dispersive Atomic Optical Bistability . 64

2.6 Experimental Demonstrations of Two-level Atomic Optical Bistability . . . . . . . . . . . . 67

2.7 Potential Applications of Atomic Optical Bistability . . . 74

3. Three-level Atoms as the Intracavity Medium and Atomic

Optical Bistability

3.1 Three-level Atoms as the Intracavity Medium _ . . . . 75

3.1.1 Cavity linewidth narrowing effect due to threelevel medium inside an optical cavity . . . . . . .

3.1.2 Enhanced cavity ring-down spectroscopy with a three-level electromagnetically induced transparency system . . . . . . . . . . . .

3.2 Atomic Optical Bistability with Three-level Atomic System 
3.2.1 Equations for three-level atomic dynamics and field propagation . . . . . . . . . .

3.2.2 Experiments on controlling the steady-state shape and thresholds of the atomic optical bistability . . 103

3.2.3 Experimental control of the rotating direction of the hysteresis cycle of atomic optical bistability . 110

4. Atomic Optical Multistability

4.1 Atomic Optical Multistability: Introduction . . . . . . . . 115

4.2 Atomic Optical Multistability with Three-level Atoms inside an Optical Cavity . . . . . . . . . . . . . . . . 116

4.2.1 Theoretical calculation . . . . . . . . . . . 116

4.2.2 Experimental demonstration of optical multistability . . . . . . . . . . . 120

4.3 Mixed Absorptive and Dispersive Effects in Atomic Optical Multistability . . . . . . . . . . . . . 123

5. Dynamical Instability to Chaos 127

5.1 Simple Model . . . . . . . . . . . . . . . . . . 127

5.2 Dynamics of a Coupled Cavity and Two-level Atomic System . . . . . . . . . . . . . . 129

5.2.1 Theoretical model of dynamical instability in a two-level system . . . . . . . . . . . . . 130

5.2.2 Experimental demonstrations of single-mode instability in the two-level system . . . . . . . . . . 135

5.3 Dynamical Instabilities in Three-level Atomic Optical Bistability . . . . . . . . . . . . . 138

5.3.1 Experimental demonstration of instability in a three-level EIT medium . . . . . . . . . . . . . . . 139

5.3.2 Theoretical calculation . . . . . . . . . . . 143

5.4 Chaotic Dynamics in the Three-level Atomic System . . . 148

5.4.1 Observation of period-doubling to chaos . . . . . . 149

5.4 .2 Theoretical calculation . . . . . . . . . . . 151

6. Controlled All-optical Switching 157

$6.1 \quad$ All-optical Switching . . . . . . . . . . . . . . . . 157

6.2 All-optical Switching in the Three-level AOB System . . . 160 
6.2.1 Deterministic switching: controlled switching between bistable states . . . . . . . . . . . 161

6.3 Controlling Light with Light in the Three-level AOB System . . . . . . . . . . . . . . . . . 164

6.4 Controlling Cavity Output with Coupling Beam Frequency 169

7. Dynamical Hysteresis and Noise-induced Behaviors 175

7.1 Dynamical Hysteresis Cycle . . . . . . . . . . . . . . . 175

7.1.1 Theoretical model . . . . . . . . . . . . . . . 176

7.1.2 Experimental results . . . . . . . . . . . . 180

7.2 Stochastic Resonance in Atomic Optical Bistability . . . . 183

7.2.1 General theoretical model . . . . . . . . . . . 185

7.2.2 Experimental demonstration of stochastic resonance .................. 187

7.3 Noise-induced Switching between Bistable States in a

Three-level Atomic Optical Bistability System . . . . . . . 192

7.3.1 Basic theory of noise-induced switching . . . . 193

7.3.2 Experimental studies of noise-induced switching . 198

8. Conclusion and Outlooks 205

$\begin{array}{ll}\text { Appendix A } & 209\end{array}$

A.1 Model of a Nonlinear Oscillator for Optical Bistability . . 209

$\begin{array}{ll}\text { Bibliography } & 215\end{array}$

$\begin{array}{ll}\text { Index } & 227\end{array}$ 